\title{
On Generating Orthogonal Polynomials for Discrete Measures
}

\author{
H.-J. Fischer
}

\begin{abstract}
In the present paper, we derive an algorithm for computing the recurrence coefficients of orthogonal polynomials with respect to discrete measures. This means that the support of the measure is a finite set. The algorithm is based on formulae of Nevai describing the transformation of recurrence coefficients, if we add a point mass to the measure of orthogonality (generalized to the case of discrete measures). The numerical condition of the problem as well as the stability properties of the new algorithm and of the well-known Stieltjes-procedure are investigated. As examples show, this new algorithm has stability properties similar to procedures based on the Lanczos algorithm.
\end{abstract}

Keywords: Orthogonal polynomials, Stieltjes procedure

AMS subject classification: $42 \mathrm{C} 05$

\section{Introduction}

Let $\sigma$ be a given positive measure with infinite support $S(\sigma)$ and finite moments of all orders. Then there exists a unique family of monic polynomials $\tilde{\pi}_{j}$ with

$$
\int \tilde{\pi}_{l}(x) \tilde{\pi}_{j}(x) d \sigma(x)=0 \quad(l<j) \text { and } \int \tilde{\pi}_{j}^{2}(x) d \sigma(x)=\gamma_{j}^{-2}>0(j \geq 0)
$$

( $\gamma_{j}$ is the leading coefficient of the orthonormal polynomial of degree $j$ ). They satisfy a three-term recurrence relation

$$
\tilde{\pi}_{j+1}(x)=\left(x-\alpha_{j}\right) \tilde{\pi}_{j}(x)-\beta_{j} \tilde{\pi}_{j-1}(x) \quad(j \geq 0)
$$

if we set $\tilde{\pi}_{-1}(x) \equiv 0$. The value of the coefficient $\beta_{0}$ has no meaning for the recurrence relation.

If the support $S(\sigma)$ is a finite set $\left\{\tau_{1}, \ldots, \tau_{N}\right\}$, the situation changes (compare the discussion in [8: Introduction] - Gautschi calls this a discrete measure): The family of monic polynomials satisfying the orthogonality conditions ( 1 ) is also finite. It consists of $N$ polynomials $\tilde{\pi}_{0}, \ldots, \tilde{\pi}_{N-1}$. Formally we can calculate from the recurrence relation (2) one more polynomial $\tilde{\pi}_{N}$, but we will have $\int \tilde{\pi}_{N}^{2}(x) d \sigma(x)=0$ (see Section 3 below).

As Gautschi [8] explained, the problem of generating orthogonal polynomials is equivalent to the problem of calculating the coefficients $\alpha_{j}$ and $\beta_{j}$ in their basic recurrence relation (2). In principle, this task always can be reduced to the case of a discrete measure. To make this more precise, we introduce some notations.

H.-J. Fischer: 'Technische Universität Chemıitz, Fakultät für Mathematik, D - 09107 Chemnitz 
Definition 1. The vector of the first recursion coefficients

$$
\left[\dot{\alpha}_{0}, \ldots, \alpha_{n-1}, \beta_{1}, \ldots, \beta_{n-1}\right]^{T}
$$

will be denoted by $\mathbf{b}_{n}$ (or by $\mathbf{b}_{n}(\sigma)$ to indicate the dependence on the measure).

Definition 2. For some real $t$ let $\delta_{t}$ be the discrete measure defined by

$$
\int f(x) d \delta_{t}(x)=f(t)
$$

for any function $f$ (this is just a point mass of weight 1 at $t$ ).

Definition 3. The set of all polynomials of degree $\leq d$ will be denoted by $\mathcal{P}_{d}$.

Definition 4. We say that the discrete measure $\sigma_{N}=\sum_{i=1}^{N} \lambda_{i} \delta_{r_{i}}$ is a discretization of the measure $\sigma$ of algebraic precision $d$ if

$$
\int q(x) d \dot{d}(x)=\int q(x) d \sigma_{N}(x)=\sum_{i=1}^{N} \lambda_{i} q\left(\tau_{i}\right),
$$

for any $q \in \mathcal{P}_{d}$.

Now it is known that the coefficients $b_{n}(\sigma)$ depend only on the moments of order up to $2 n-1$ (see equations (4) and (5) below) and thus the recursion coefficients $b_{n}(\sigma)$ and $\mathbf{b}_{n}\left(\sigma_{N}\right)$ for the measure $\sigma$ and the discretization $\sigma_{N}$ of algebraic precision $\geq 2 n-1$ coincide. But discretizations of sufficiently high algebraic precision trivially exist: If $\tau_{1}, \ldots, \tau_{N}$ and $\lambda_{1}, \ldots, \lambda_{N}$ are the nodes and weights of a Gauss-Christoffel quadrature formula, respectively, then $\sigma_{N}$ will have algebraic precision $2 N-1$. In practice, of course, the Gauss-Christoffel rule for some measure $\sigma$ may be unknown, but discretizations of sufficiently high algebraic precision are often available.

Usually, the coefficients $\mathbf{b}_{n}\left(\sigma_{N}\right)$ for discrete measures $\sigma_{N}$ are calculated with the well-known Stieltjes procedure, which we analyze in the next section. Paradoxically, the method is reliable in many cases where the discretization $\sigma_{N}$ is only an approximation to a continuous measure $\sigma$. But it breaks down completely in some cases where the measure itself is discrete or has discrete components! A striking example for this situation is Gautschi's Example 4.8 in [8: p. 315]: He adds a point mass of weight 1 at $x=2$ to the absolutely continuous distribution $\sigma$ with $d \sigma(x)=d x$ on $[-1,-0.5] \cup[0.5,1]$. The Stieltjes procedure is not suitable to calculate accurately the first coefficients $\mathbf{b}_{n}\left(\sigma+\delta_{2}\right)$ already for rather small values of $n$. Gautschi reports an error growth of more than $4 \cdot 10^{14}$ for $n=24$ and remarks: "We know of no stable method to compute orthogonal polynomials of the type introduced in Example 4.8." Fortunately, the situation has changed. Gautschi retracted this statement in his 1994 paper [12]. In the ORTHPOLpackage of Gautschi, a routine lancz is included, which is based on the Rutishauser stabilization of the diagonal Hermitian Lanczos process (see the article of Gragg and Harrod [13] or the generalization of Reichel [17]). This algorithm is fast and stable, but it is derived and formulated in the language of inverse spectral problems. However, there is a simple derivation of a procedure completely in the language of discrete measures and orthogonal polynomials. 
In Nevai's famous memoir [16], he has shown how to systematically add mass points to measures. This was known earlier for special systems of orthogonal polynomials. Adding some details, we are able to derive a new algorithm which is more stable than Stieltjes procedure and comparable to lancz.

\section{Stieltjes procedure and its stability}

The cocfficients $\alpha_{j}$ and $\beta_{j}$ can be expressed by simple formulas (see [8: Equation (2.2)]): We have

$$
\alpha_{j}=\frac{\int x \tilde{\pi}_{j}^{2}(x) d \sigma(x)}{\int \tilde{\pi}_{j}^{2}(x) d \sigma(x)} \quad(j \geq 0)
$$

and

$$
\beta_{j}=\frac{\int \tilde{\pi}_{j}^{2}(x) d \sigma(x)}{\int \tilde{\pi}_{j-1}^{2}(x) d \sigma(x)} \quad(j . \geq 1)
$$

These formulas together with the recurrence relation (2) can be used to calculate recursively as many coefficients $\alpha_{j}$ and $\beta_{j}$ (and polynomials $\tilde{\pi}_{j}$ ) as desired (compare Gautschi [8: p. 292] or (10: p. 198]): Starting from $\tilde{\pi}_{-1}=0$ and $\tilde{\pi}_{0}=1$ we can compute $\alpha_{0}$ from (4) with $j=0$. Now (2) gives the new polynomial $\tilde{\pi}_{1}$ allowing to compute $\alpha_{1}$ from (4) and $\beta_{1}$ from (5).

At step $k$ of this algorithm we have calculated $\mathbf{b}_{k}$. Then we use the recurrence relation (2) with $j=k-1$ to compute $\tilde{\pi}_{k}$ and from this (via (4) and (5) with $j=k$ ) we obtain the new coefficients $\alpha_{k}$ and $\beta_{k}$.

As was mentioned by Gautschi [10: p. 198], in order to implement this algorithm we have to find an appropriate codification of the polynomials $\tilde{\pi}_{j}$ allowing the evaluation of the integrals in (4) and (5). If the given measure is discrete or has a discretization $\sigma_{N}=\sum_{i=1}^{N} \lambda_{i} \delta_{T_{i}}$ of sufficiently high algebraic precision, then we can use equation (3) to compute the integrals. In this case, the polynomial $\tilde{\pi}_{j}$ will be represented by the vector $\left[\tilde{\pi}_{j}\left(\tau_{1}\right), \ldots, \tilde{\pi}_{j}\left(\tau_{N}\right)\right]^{T}$. In this form the algorithm is called (discretized) Stieltjes procedure.

The stability of the algorithm depends on the stability of the recurrence relation (2). Though we are not able to analyze this difficult question completely, we can say that the recurrence (2) is unstable in isolated mass points $x$ of the measure $\sigma$. We will give rough estimates for the order of magnitude of the resulting error magnification (compare the similar analysis in the paper of Gautschi [11]).

We start from the well-known fact that instability of the recurrence relation (2) is caused by the introduction (via rounding errors) of another independent solution of the recursive equation. Thus we compute not $\tilde{\pi}_{j}$, but $\tilde{\pi}_{j}+\varepsilon \tilde{\rho}_{j}$, where $\varepsilon$ is of the order of the machine eps and $\tilde{\rho}_{j}$ are the numerator polynomials (see Appendix). Consequently, instead of the quantities $\int \tilde{\pi}_{j}^{2}(x) d \sigma(x)$ and $\int x \tilde{\pi}_{j}^{2}(x) d \sigma(x)$ occuring in equations (4) and (5) we calculate

$$
\int\left(\tilde{\pi}_{j}(x)+\varepsilon \dot{\rho}_{j}(x)\right)^{2} d \sigma(x)=\int \tilde{\pi}_{j}^{2}(x) d \sigma(x)+\varepsilon^{2} \int \tilde{j}_{j}^{2}(x) d \sigma(x)
$$


and

$$
\int x\left(\tilde{\pi}_{j}(x)+\varepsilon \tilde{\rho}_{j}(x)\right)^{2} d \sigma(x)=\int x \tilde{\pi}_{j}^{2}(x) d \sigma(x)+2 \varepsilon \gamma_{j}^{-2}+\varepsilon^{2} \int x \tilde{\rho}_{j}^{2}(x) d \sigma(x)
$$

(observe that $\tilde{\rho}_{j} \in \mathcal{P}_{j-1}$ and that $x \tilde{\rho}_{j}(x)$ is a monic polynomial contained in $\mathcal{P}_{j}$ ). Since we consider measures $\sigma$ with bounded support $S(\sigma)$, the error in the recurrence coefficients $\alpha_{j}$ and $\beta_{j}$ is characterized by

$$
E_{j}=\varepsilon^{2} \frac{\int \tilde{\rho}_{j}^{2}(x) d \sigma(x)}{\int \tilde{\pi}_{j}^{2}(x) d \sigma(x)}=\varepsilon^{2} \gamma_{j}^{2} \int \tilde{\rho}_{j}^{2}(x) d \sigma(x)
$$

In the Appendix we will show that the numerator polynomials may grow exponentially with their degree in isolated mass points of the measure $\sigma$ (see Lemma 12 below).

Proposition 1. If $\tau$ is a mass point with weight $\lambda$, we can expect an error magni. fication $M_{n}=E_{n} / \varepsilon$ in the coefficients $\mathrm{b}_{n}$ that satisfies $M_{n} \geq \varepsilon \lambda \gamma_{n}^{2} \tilde{\rho}_{n}^{2}(\tau)$.

In contrast to this, if the support of the measure has some regularity property, then the error magnification grows only polynomially with the degree $n$ (see Lemma 8 in the Appendix).

\section{Adding point masses}

Nevai [16] formulated his results in terms of the orthonormal polynomials $\pi_{j}$. Their orthogonality relation reads

$$
\int \pi_{l}(x) \pi_{j}(x) d \sigma(x)=0(l<j) \quad \text { and } \quad \int \pi_{j}^{2}(x) d \sigma(x)=1
$$

(for $j \leq N-1$ if the support of $\sigma$ contains $N$ points only). They are uniquely determined if we assume the highest coefficient $\gamma_{j}$ in $\pi_{j}$ to be positive. Obviously, we have $\pi_{j}=\gamma_{j} \tilde{\pi}_{j}$ and from (6) $\gamma_{j}^{-2}=\int \tilde{\pi}_{j}^{2}(x) d \sigma(x)$. This gives (see (5))

$$
\gamma_{j}^{2}=\frac{\gamma_{j-1}^{2}}{\beta_{j}}
$$

for $j \geq 1$ (note that Nevai [16] always writes $\gamma_{j-1} / \gamma_{j}$ instead of $\beta_{j}^{1 / 2}$ ). The recurrence relation for these polynomials is

$$
\beta_{j+1}^{1 / 2} \pi_{j+1}(x)=\left(x-\alpha_{j}\right) \pi_{j}(x)-\beta_{j}^{1 / 2} \pi_{j-1}(x)
$$

for $j \geq 0$ (and $j \leq N-2$ if $S(\sigma)$ has just $N$ elements).

Now we are able to formulate Nevai's lemma [16: Lemma 7.15] in our notations (we simply write $\alpha_{j}, \beta_{j}, \ldots$ instead of $\left.\alpha_{j}(\sigma), \beta_{j}(\sigma), \ldots\right)$. 
Lemma 1. Let $\sigma$ be a measure, $\pi_{j}$ the orthonormal polynomials with respect to this measure satisfying the recurrence relation (8) and $\nu=\sigma+\lambda \delta_{\tau}$. Then the recurrence coefficients for the orthogonal polynomials with respect to $\nu$ can be computed from

$$
\alpha_{j}(\nu)=\alpha_{j}+\lambda \frac{\beta_{j+1}^{1 / 2} \pi_{j}(\tau) \pi_{j+1}(\tau)}{1+\lambda \sum_{i=0}^{j} \pi_{i}^{2}(\tau)}-\lambda \frac{\beta_{j}^{1 / 2} \pi_{j}(\tau) \pi_{j-1}(\tau)}{1+\lambda \sum_{i=0}^{j-1} \pi_{i}^{2}(\tau)}
$$

and

$$
\beta_{j}(\nu)=\beta_{j} \frac{\left[1+\lambda \sum_{i=0}^{j-2} \pi_{i}^{2}(\tau)\right]\left[1+\lambda \sum_{i=0}^{j} \pi_{i}^{2}(\tau)\right]}{\left[1+\lambda \sum_{i=0}^{j-1} \pi_{i}^{2}(\tau)\right]^{2}}
$$

For computational purposes, it is better to work with monic polynomials in order to avoid square roots in (8) and (9). Moreover, if we apply the lemma to a discrete measure $\sigma=\sum_{i=1}^{N} \lambda_{i} \delta_{\tau_{i}}$, there is a difficulty: if all $\tau_{i}$ and $\tau$ are different and all $\lambda_{i}$ and $\lambda$ are positive (i.e. we have really $N+1$ mass points), then $\alpha_{N}(\nu)$ is defined, but we can not compute it from (9), since $\alpha_{N}(\dot{\sigma})$ is not defined.

For these reasons we rederive the relcvant equations here. First, we need some auxiliary results.

Lemma 2. The coefficient of $x^{n-1}$ in $\tilde{\pi}_{n}(x)$ is $-\sum_{j=0}^{n-1} \alpha_{j}$.

This is just [3: Chapter I/Theorem 4.2(d)]. Now we are able to prove a simple relation for all existing recurrence coefficients $\alpha_{j}$ of a discrete measure.

Lemma 3. Let $\sigma=\sum_{i=1}^{N} \lambda_{i} \delta_{r_{i}}$ be a discrete measure, where $\lambda_{i}>0$ for $i=1, \ldots, N$ and all $\tau_{i}$ are different. Then $\sum_{j=0}^{N-1} \alpha_{j}=\sum_{i=1}^{N} \tau_{i}$.

Proof. We prove first that

$$
\tilde{\pi}_{N}(x)=\left(x-\tau_{1}\right) \cdots\left(x-\tau_{N}\right) .
$$

Indeed, both polynomials of the same degree $N$ are monic, and so its difference $r$ is in $\mathcal{P}_{N-1}$. Consequently, $r$ is orthogonal to $\tilde{\pi}_{N}$. But $r$ is also orthogonal to the polynomial $\left(x-\tau_{1}\right) \cdots\left(x-\tau_{N}\right)$. Thus $r$ must be orthogonal to itself, i.e.

$$
\int r^{2}(x) d \sigma(x)=\sum_{i=1}^{N} \lambda_{i} r^{2}\left(\tau_{i}\right)=0
$$

From this we see that $r\left(\tau_{i}\right)=0$ for $i=1, \ldots, N$. Now $r \in \mathcal{P}_{N-1}$ implies $r \equiv 0$. Comparing the leading coefficients of both sides in equation (10), we immediately obtain our proposition: The coefficient of $x^{N-1}$ in $\tilde{\pi}_{N}(x)$ is $-\sum_{j=0}^{N-1} \alpha_{j}$ (see Lemma 2), and the same coefficient in the right-hand side is $-\sum_{i=1}^{N} \tau_{i}$, thus our proposition is true

After these preparations we can formulate the main result. 
Theorem 1. Let $\sigma=\sum_{i=1}^{N} \lambda_{i} \delta_{\tau_{i}}$ be a discrete measure, where $\lambda_{i}>0$ for $i=$ $1, \ldots, N$ and all $\tau_{i}$ are different. Further, let $\lambda>0, \tau$ be different from all $\tau_{i}$, and $\nu=\sigma+\lambda \delta_{r}$. Then we have

$$
\alpha_{j}(\nu)=\alpha_{j}+\lambda \frac{\gamma_{j}^{2} \tilde{\pi}_{j+1}(\tau) \tilde{\pi}_{j}(\tau)}{1+\lambda \sum_{i=0}^{j} \gamma_{i}^{2} \tilde{\pi}_{i}^{2}(\tau)}-\lambda \frac{\gamma_{j-1}^{2} \tilde{\pi}_{j}(\tau) \tilde{\pi}_{j-1}(\tau)}{1+\lambda \sum_{i=0}^{j-1} \gamma_{i}^{2} \tilde{\pi}_{i}^{2}(\tau)}
$$

and

$$
\beta_{j}(\nu)=\beta_{j} \frac{\left[1+\lambda \sum_{i=0}^{j-2} \gamma_{i}^{2} \tilde{\pi}_{i}^{2}(\tau)\right]\left[1+\lambda \sum_{i=0}^{j} \gamma_{i}^{2} \tilde{\pi}_{i}^{2}(\tau)\right]}{\left[1+\lambda \sum_{i=0}^{j-1} \gamma_{i}^{2} \tilde{\pi}_{i}^{2}(\tau)\right]^{2}}
$$

for $j<N$, and furthermore

$$
\alpha_{N}(\nu)=\tau-\lambda \frac{\gamma_{N-1}^{2} \tilde{\pi}_{N}(\tau) \tilde{\pi}_{N-1}(\tau)}{1+\lambda \sum_{i=0}^{N-1} \gamma_{i}^{2} \tilde{\pi}_{i}^{2}(\tau)}
$$

and

$$
\beta_{N}(\nu)=\frac{\lambda \gamma_{N-1}^{2} \tilde{\pi}_{N}^{2}(\tau)\left[1+\lambda \sum_{i=0}^{N-2} \gamma_{i}^{2} \tilde{\pi}_{i}^{2}(\tau)\right]}{\left[1+\lambda \sum_{i=0}^{N-1} \gamma_{i}^{2} \tilde{\pi}_{i}^{2}(\tau)\right]^{2} \ldots}
$$

Proof. We denote the monic orthogonal polynomials with respect to $\nu$ by $p_{j}$. Then we must have

$$
p_{j}(x)=\tilde{\pi}_{j}(x)+\sum_{l<j} c_{j l} \tilde{\pi}_{l}(x)
$$

with some unknown coefficients $c_{j l}$. Multiplying this by $\tilde{\pi}_{l}(x)$ und integrating we obtain

$$
0=\int p_{j}(x) \tilde{\pi}_{l}(x) d \nu(x)=\int p_{j}(x) \tilde{\pi}_{l}(x) d \sigma(x)+\lambda p_{j}(\tau) \tilde{\pi}_{l}(\tau)=c_{\jmath} l \gamma_{l}^{-2}+\lambda \dot{p}_{j}(\tau) \tilde{\pi}_{l}(\tau)
$$

and from this $c_{j l}=-\lambda \gamma_{l}^{2} p_{j}(\tau) \tilde{\pi}_{l}(\tau)$. Putting the last result into (15) for $x=\tau$ and then solving for $p_{j}(\tau)$, we finally obtain

$$
p_{j}(r)=\frac{\tilde{\pi}_{j}(\tau)}{1+\lambda \sum_{i=0}^{j-1} \gamma_{i}^{2} \tilde{\pi}_{i}^{2}(\tau)}
$$

and

$$
c_{j l}=-\frac{\lambda \gamma_{l}^{2} \tilde{\pi}_{j}(\tau) \tilde{\pi}_{l}(\tau)}{1+\lambda \sum_{i=0}^{j-1} \gamma_{i}^{2} \tilde{\pi}_{i}^{2}(\tau)}
$$


Comparing the coefficients of $x^{j-1}$ in both sides of (15) we have by virtue of Lemma 2

$$
-\sum_{i=0}^{j-1} \alpha_{i}(\nu)=-\sum_{i=0}^{j-1} \alpha_{i}+c_{j j-1}=-\sum_{i=0}^{j-1} \alpha_{i}-\frac{\lambda \gamma_{j-1}^{2} \tilde{\pi}_{j}(\tau) \tilde{\pi}_{j-1}(\tau)}{1+\lambda \sum_{i=0}^{j-1} \gamma_{i}^{2} \tilde{\pi}_{i}^{2}(\tau)} \quad(j \leq N) .
$$

Taking differences we immediately arrive at (11) for $j+1 \leq N$. The last coefficient $\alpha_{N}(\nu)$ can be calculated from Lemma 3. Applying the lemma to the measures $\sigma$ and $\nu$ we have

$$
\sum_{i=0}^{N-1} \alpha_{i}=\sum_{i=1}^{N} \tau_{i} \quad \text { and } \quad \sum_{i=0}^{N} \alpha_{i}(\nu)=\sum_{i=1}^{N} \tau_{i}+\tau
$$

and consequently

$$
\sum_{i=0}^{N} \alpha_{i}(\nu)=\sum_{i=0}^{N-1} \alpha_{i}+\tau .
$$

Solving for $\alpha_{N}(\nu)$ and using (18) for $j=N$ we obtain (13).

In order to calculate the coefficients $\beta_{j}(\nu)$ we use (5). From (15) we have

$$
\begin{aligned}
\int p_{j}^{2}(x) d \nu(x) & =\int p_{j}^{2}(x) d \sigma(x)+\lambda p_{j}^{2}(\tau) \\
& =\int \tilde{\pi}_{j}^{2}(x) d \sigma(x)+\sum_{l=0}^{j-1} c_{j l}^{2} \int \tilde{\pi}_{l}^{2}(x) d \sigma(x)+\lambda p_{j}^{2}(\tau)
\end{aligned}
$$

Since $\int \tilde{\pi}_{j}^{2}(x) d \sigma(x)=\gamma_{j}^{-2}(j<N)$ and $\int \tilde{\pi}_{N}^{2}(x) d \sigma(x)=0$, using (16) and (17) we get

$$
\gamma_{j}(\nu)^{-2}=\int p_{j}^{2}(x) d \nu(x)=\gamma_{j}^{-2}+\frac{\lambda \tilde{\pi}_{j}^{2}(\tau)}{1+\lambda \sum_{i=0}^{j-1} \gamma_{i}^{2} \tilde{\pi}_{i}^{2}(\tau)}=\gamma_{j}^{-2} \frac{1+\lambda \sum_{i=0}^{j} \gamma_{i}^{2} \tilde{\pi}_{i}^{2}(\tau)}{1+\lambda \sum_{i=0}^{j-1} \gamma_{i}^{2} \tilde{\pi}_{i}^{2}(\tau)}
$$

for $j<N$ and

$$
\int p_{N}^{2}(x) d \nu(x)=\frac{\lambda \tilde{\pi}_{N}^{2}(\tau)}{1+\lambda \sum_{i=0}^{N-1} \gamma_{i}^{2} \tilde{\pi}_{i}^{2}(\tau)}
$$

Now from (5) (for the measure $\nu$ ) and (7) we obtain (12) and (14)

Using equations (11) - (14) together with the recurrence relations (2) and (7) it is possible to design a new algorithm which adds point masses one by one and computes the recurrence coefficients of a discrete measure starting from nothing.

The numerical stability of this algorithm obviously depends on the stability of the recurrence relations (2), as well. If we add points in ascending order, then any new point will be outside the interval of orthogonality of the measure constructed so far, and Lemma 9 will be applicable. 


\section{Questions of numerical condition}

In this section we investigate the sensitivity of the map $H_{n}:\left[\tau_{1}, \ldots, \tau_{N}, \lambda_{1}, \ldots, \lambda_{N}\right]^{T} \rightarrow$ $\mathbf{b}_{n}$ from the nodes and weights of a discrete measure $\sigma=\sum_{i=1}^{N} \lambda_{i} \delta_{\tau_{i}}$ to the recurrence coefficients $\mathbf{b}_{n}=\mathbf{b}_{n}(\sigma)$.

This problem has an interesting history: In his paper [7] Gautschi explained that the inverse map $H_{n}^{-1}$ is essentially an eigenvalue problem for the symmetric positive definite Jacobi matrix and thus should be well-conditioned. However, no estimates for condition numbers were given. In his article [8] Gautschi gave estimates for the condition number which (if used as a-posteriori bounds!) seemed to show bad conditioning in some cases. Unfortunately, his formulas were partially erroneous, and Gautschi corrected his statement in [9: Note added in proof]. But for all we know no correct estimates of the sensitivity of the map $H_{n}$ appeared in the literature.

Our analysis is based on the following rather general result concerning dependence on a parameter.

Lemma 4. Let $l$ be some positive linear functional on $\mathcal{P}_{2 n-1}$, differentiably depending on some parameter $t$, and let the functional $c$ be defined by $\frac{\partial l}{\partial t}=c$. Then there exist unique monic polynomials $\tilde{\pi}_{0}, \ldots, \tilde{\pi}_{n-1}$ with

$$
l\left(\tilde{\pi}_{j} \tilde{\pi}_{i}\right)=0 \quad(0 \leq i<j \leq n-1) \quad \text { and } \quad l\left(\tilde{\pi}_{j}^{2}\right)=\gamma_{j}^{-2}>0 \quad(0 \leq j \leq n-1),
$$

fulfilling the recurrence relation (2). The partial derivatives of the recursion coefficients with respect to $t$ are

$$
\frac{\partial \alpha_{j}}{\partial t}=\gamma_{j}^{2} c\left(\tilde{\pi}_{j} \tilde{\pi}_{j+1}\right)-\gamma_{j-1}^{2} c\left(\tilde{\pi}_{j-1} \tilde{\pi}_{j}\right)
$$

and

$$
\frac{\partial \beta_{j}}{\partial t}=\beta_{j}\left[\gamma_{j}^{2} c\left(\tilde{\pi}_{j}^{2}\right)-\gamma_{j-1}^{2} c\left(\tilde{\pi}_{j-1}^{2}\right)\right] .
$$

Proof. The assertion concerning the existence of the polynomials and the recurrence relation is clear (see [3: Chapter I/Sections 3 and 4]). The coefficients $\alpha_{j}$ and $\beta_{j}$ can be calculated from

$$
\alpha_{j}=\frac{l\left(x \tilde{\pi}_{j}^{2}(x)\right)}{l\left(\tilde{\pi}_{j}^{2}\right)} \quad \text { and } \quad \beta_{j}=\frac{l\left(\tilde{\pi}_{j}^{2}\right)}{l\left(\tilde{\pi}_{j-1}^{2}\right)}=\frac{\gamma_{j-1}^{2}}{\gamma_{j}^{2}}
$$

(these equations are just (4) and (5) in another notation). Since our polynomials are monic, i.e. their highest coefficient 1 is independent of $t$, we have $\partial \tilde{\pi}_{j} / \partial t \in \mathcal{P}_{j-1}$. In order to calculate this polynomial, we expand it into a Fourier series:

$$
\frac{\partial \tilde{\pi}_{j}}{\partial t}=\sum_{i=0}^{j-1} c_{j i} \tilde{\pi}_{i} \quad \text { with } \quad c_{j i}=\frac{l\left(\frac{\partial \tilde{\pi}_{j}}{\partial t} \tilde{\pi}_{i}\right)}{l\left(\tilde{\pi}_{i}^{2}\right)}=\gamma_{i}^{2} l\left(\frac{\partial \tilde{\pi}_{j}}{\partial t} \tilde{\pi}_{i}\right) .
$$

These coeffcients can be obtained differentiating the orthogonality relations with respect to $t$. From the elementary identity

$$
\frac{\partial}{\partial t} l(q)=l\left(\frac{\partial q}{\partial t}\right)+\frac{\partial l}{\partial t}(q)=l\left(\frac{\partial q}{\partial t}\right)+c(q)
$$


we have

$$
0=\frac{\partial}{\partial t} l\left(\tilde{\pi}_{j} \tilde{\pi}_{i}\right)=l\left(\frac{\partial \tilde{\pi}_{j}}{\partial t} \tilde{\pi}_{i}\right)+l\left(\tilde{\pi}_{j} \frac{\partial \tilde{\pi}_{i}}{\partial t}\right)+c\left(\tilde{\pi}_{j} \tilde{\pi}_{i}\right) \quad(i<j)
$$

but $l\left(\tilde{\pi}_{j} \frac{\partial \tilde{\pi}_{i}}{\partial t}\right)=0$ due to orthogonality. Thus we have $c_{j i}=-\gamma_{i}^{2} c\left(\tilde{\pi}_{j} \tilde{\pi}_{i}\right)$ and consequently

$$
\frac{\partial \tilde{\pi}_{j}}{\partial t}=-\sum_{i=0}^{j-1} \gamma_{i}^{2} c\left(\tilde{\pi}_{j} \tilde{\pi}_{i}\right) \tilde{\pi}_{i}
$$

From $l\left(\tilde{\pi}_{j}^{2}\right)=\gamma_{j}^{-2}$ and $(23)$ we obtain

$$
\frac{\partial \gamma_{j}^{-2}}{\partial t}=l\left(2 \tilde{\pi}_{j} \frac{\partial \tilde{\pi}_{j}}{\partial t}\right)+c\left(\tilde{\pi}_{j}^{2}\right)=c\left(\tilde{\pi}_{j}^{2}\right)
$$

due to orthogonality again (recall $\partial \tilde{\pi}_{j} / \partial t \in \mathcal{P}_{j-1}$ ). Now equation (21) is an immediate consequence of (22) and the last equation.

By virtue of Lemma 2, the coefficient of $x^{j-1}$ in $\tilde{\pi}_{j}$ is $-\sum_{i=0}^{j-1} \alpha_{i}$. Consequently, the coefficient of $x^{j-1}$ in $\partial \tilde{\pi}_{j} / \partial t$ is $-\frac{\partial}{\partial t} \sum_{i=0}^{j-1} \alpha_{i}=-\gamma_{j-1}^{2} c\left(\tilde{\pi}_{j} \tilde{\pi}_{j-1}\right)$ due to equation (24). Taking differences we arrive at equation (20)

Remark. The idea of this proof is not new. In the article [5] we analyzed the dependence on modified moments with essentially the same method (compare 15: Theorem 1], where the result is formulated in terms of orthonormal polynomials, however). The method can be refined to be applicable to scalar products of Sobolev type, but this is beyond the scope of the present paper.

Now we are able to calculate the partial derivatives of the coefficients $\alpha_{j}$ and $\beta_{j}$ with respect to a single node or weight.

Lemma 5. Let $\sigma$ be a measure not depending on $\lambda$ and $\tau$ and let $\nu=\sigma+\lambda \delta_{r}$. We denote the monic orthogonal polynomials with respect to $\nu$ by $p_{j}$ (as in the proof of Theorem 1). Then the formulas

$$
\begin{aligned}
\frac{\partial \alpha_{j}(\nu)}{\partial \lambda}= & \gamma_{j}^{2}(\nu) p_{j}(\tau) p_{j+1}(\tau)-\gamma_{j-1}^{2}(\nu) p_{j-1}(\tau) p_{j}(\tau) \\
\frac{\partial \beta_{j}(\nu)}{\partial \lambda}= & \beta_{j}(\nu)\left[\gamma_{j}^{2}(\nu) p_{j}^{2}(\tau)-\gamma_{j-1}^{2}(\nu) p_{j-1}^{2}(\tau)\right] \\
\frac{\partial \alpha_{j}(\nu)}{\partial \tau}= & \lambda \gamma_{j}^{2}(\nu)\left[p_{j}^{\prime}(\tau) p_{j+1}(\tau)+p_{j}(\tau) p_{j+1}^{\prime}(\tau)\right] \\
& -\lambda \gamma_{j-1}^{2}(\nu)\left[p_{j-1}^{\prime}(\tau) p_{j}(\tau)+p_{j-1}(\tau) p_{j}^{\prime}(\tau)\right] \\
\frac{\partial \beta_{j}(\nu)}{\partial \tau}= & 2 \lambda \beta_{j}(\nu)\left[\gamma_{j}^{2}(\nu) p_{j}^{\prime}(\tau) p_{j}(\tau)-\gamma_{j-1}^{2}(\nu) p_{j-1}^{\prime}(\tau) p_{j-1}(\tau)\right]
\end{aligned}
$$

hold.

Proof. The proposition is an almost trivial consequence of our Lemma 4 . The linear functional $l$ is defined via $l(q)=\int q(x) d \nu(x)=\int q(x) d \sigma(x)+\lambda q(\tau)$ for any polynomial $q$, and obviously we have $\frac{\partial l}{\partial \lambda}(q)=q(\tau)$ and $\frac{\partial l}{\partial \tau}(q)=\lambda q^{\prime}(\tau)$ 
Now we are going to define an appropriate condition number cond $H_{n}$. As in [8: Section 3.1] we consider each $\alpha_{j}$ and $\beta_{j}$ individually as functions of one particular $\lambda_{i}$ or $\tau_{i}$. However, we feel that the relative error is an adequate measure for $\beta_{j}$ or $\lambda_{i}$ only. In contrast to this, some node $\tau_{i}$ and ever all coefficients $\alpha_{j}$ can be zero. Thus we base our condition number on the relative error of $\beta_{j}$ and $\lambda_{i}$ and on the absolute error of $\alpha_{j}$ and $\tau_{i}$, i.e.

$$
\begin{array}{ll}
\left(\operatorname{cond} \alpha_{j}\right)\left(\lambda_{i}\right)=\left|\lambda_{i} \frac{\partial \alpha_{j}}{\partial \lambda_{i}}\right| & (j=0, \ldots, n-1 ; i=1, \ldots, N) \\
\left(\operatorname{cond} \alpha_{j}\right)\left(\tau_{i}\right)=\left|\frac{\partial \alpha_{j}}{\partial \tau_{i}}\right| & (j=0, \ldots, n-1 ; i=1, \ldots, N) \\
\left(\operatorname{cond} \beta_{j}\right)\left(\lambda_{i}\right)=\left|\beta_{j}^{-1} \lambda_{i} \frac{\partial \beta_{j}}{\partial \lambda_{i}}\right| & (j=1, \ldots, n-1 ; i=1, \ldots, N) \\
\left(\operatorname{cond} \beta_{j}\right)\left(\tau_{i}\right)=\left|\beta_{j}^{-1} \frac{\partial \beta_{j}}{\partial \tau_{i}}\right| & (j=1, \ldots, n-1 ; i=1, \ldots, N) .
\end{array}
$$

The sensitivity of a single coefficient $\alpha$; or $\beta_{j}$ to errors in the initial data can now be measured by

$$
\begin{array}{ll}
\operatorname{cond} \alpha_{j}=\sum_{i=1}^{N}\left[\left(\operatorname{cond} \alpha_{j}\right)\left(\lambda_{i}\right)+\left(\operatorname{cond} \alpha_{j}\right)\left(\tau_{i}\right)\right] & (j=0, \ldots, n-1) \\
\operatorname{cond} \beta_{j}=\sum_{i=1}^{N}\left[\left(\operatorname{cond} \beta_{j}\right)\left(\lambda_{i}\right)+\left(\operatorname{cond} \beta_{j}\right)\left(\tau_{i}\right)\right] & (j=1, \ldots, n-1) .
\end{array}
$$

A suitable condition number is the maximum over all these quantities:

$$
\operatorname{cond} H_{n}=\max \left\{\max _{j=0, \ldots, n-1} \operatorname{cond} \alpha_{j}, \max _{j=1, \ldots, n-1} \operatorname{cond} \beta_{j}\right\} \text {. }
$$

The following Theorem 2 is an immediate consequence of equations (25) -.(28) above.

Theorem 2. Let $\sigma=\sum_{i=1}^{N} \lambda_{i} \delta_{r_{i}}$ be a discrete measure, $b_{n}$ the recurrence coeffi. cients, $\gamma_{j}^{2}$ the normalization constants and $\tilde{\pi}_{j}$ the monic orthogonal polynomials with respect to $\sigma$. Then we have

$$
\begin{aligned}
& \text { cond } H_{n}=\max \{ \\
& \max _{j=0, \ldots, n-1} \sum_{i=1}^{N} \lambda_{i} \gamma_{j}^{2}\left(\left|\tilde{\pi}_{j}\left(\tau_{i}\right) \tilde{\pi}_{j+1}\left(\tau_{i}\right)-\beta_{j} \tilde{\pi}_{j-1}\left(\tau_{i}\right) \tilde{\pi}_{j}\left(\tau_{i}\right)\right|\right. \\
&\left.+\left|\left[\tilde{\pi}_{j}^{\prime}\left(\tau_{i}\right) \tilde{\pi}_{j+1}\left(\tau_{i}\right)+\tilde{\pi}_{j}\left(\tau_{i}\right) \tilde{\pi}_{j+1}^{\prime}\left(\tau_{i}\right)\right]-\beta_{j}\left[\tilde{\pi}_{j-1}^{\prime}\left(\tau_{i}\right) \tilde{\pi}_{j}\left(\tau_{i}\right)+\tilde{\pi}_{j-1}\left(\tau_{i}\right) \tilde{\pi}_{j}^{\prime}\left(\tau_{i}\right)\right]\right|\right) \\
&\left.\max _{j=1, \ldots, n-1} \sum_{i=1}^{N} \lambda_{i} \gamma_{j}^{2}\left(\left|\tilde{\pi}_{j}^{2}\left(\tau_{i}\right)-\beta_{j} \tilde{\pi}_{j-1}^{2}\left(\tau_{i}\right)\right|+2\left|\tilde{\pi}_{j}^{\prime}\left(\tau_{i}\right) \tilde{\pi}_{j}\left(\tau_{i}\right)-\beta_{j} \tilde{\pi}_{j-1}^{\prime}\left(\tau_{i}\right) \tilde{\pi}_{j-1}\left(\tau_{i}\right)\right|\right)\right\}
\end{aligned}
$$

Proof. The proof is just by insertion of equations (25) - (28) into the above definitions (recall $\gamma_{j-1}^{2}=\beta_{j} \gamma_{j}^{2}$ ) 
However, these formulas are applicable only as a-priori bounds. If instead we use the results of some unstable algorithm, the obtained figures will show this instability, but not the conditioning of our problem. Some relevant a-priori estimates of the size of orthogonal polynomials and their derivatives are summarized in the Appendix. We can use them to obtain the following results.

Corollary 1. Let $\sigma_{N}=\sum_{i=1}^{N} \lambda_{i} \delta_{\tau_{i}}$ be a discretization of algebraic precision $M$ of some measure $\sigma$ satisfying (34) with support $S(\sigma) \in \mathcal{M}^{2}(C, \alpha)$ (see Definition 6). Then the condition number of our problem can be estimated by

$$
\text { cond } H_{n} \leq C_{1}(C, \alpha) n^{\alpha} \quad \text { for } 2 n \leq M \text {, }
$$

where $C_{1}(C, \alpha)$ denotes a constant depending on $C$ and $\alpha$.

Proof. We simply estimate the sums in Theorem 2 using the Cauchy-Schwarz inequality. The sums not including derivatives are uniformly bounded, if we assume $S(\sigma) \subset[a, b]:$

$$
\begin{aligned}
& \sum_{i=1}^{N} \lambda_{i} \gamma_{j}^{2}\left|\tilde{\pi}_{j}\left(\tau_{i}\right) \tilde{\pi}_{j+1}\left(\tau_{i}\right)-\beta_{j} \tilde{\pi}_{j-1}\left(\tau_{i}\right) \tilde{\pi}_{j}\left(\tau_{i}\right)\right| \\
& \quad \leq \sum_{i=1}^{N} \lambda_{i} \gamma_{j}^{2}\left|\tilde{\pi}_{j}\left(\tau_{i}\right) \tilde{\pi}_{j+1}\left(\tau_{i}\right)\right|+\sum_{i=1}^{N} \lambda_{i} \gamma_{j}^{2} \beta_{j}\left|\tilde{\pi}_{j-1}\left(\tau_{i}\right) \tilde{\pi}_{j}\left(\tau_{i}\right)\right| \\
& \quad \leq \gamma_{j}^{2}\left(\sqrt{\sum_{i=1}^{N} \lambda_{i} \tilde{\pi}_{j}^{2}\left(\tau_{i}\right) \sum_{i=1}^{N} \lambda_{i} \tilde{\pi}_{j+1}^{2}\left(\tau_{i}\right)}+\beta_{j} \sqrt{\sum_{i=1}^{N} \lambda_{i} \tilde{\pi}_{j-1}^{2}\left(\tau_{i}\right) \sum_{i=1}^{N} \lambda_{i} \tilde{\pi}_{j}^{2}\left(\tau_{i}\right)}\right) \\
& \quad=\gamma_{j}^{2}\left(\left\|\tilde{\pi}_{j}\right\|_{2}\left\|\tilde{\pi}_{j+1}\right\|_{2}+\beta_{j}\left\|\tilde{\pi}_{j-1}\right\|_{2}\left\|\tilde{\pi}_{j}\right\|_{2}\right) \\
& \quad=\beta_{j+1}^{1 / 2}+\beta_{j}^{1 / 2} \\
& \leq b-a
\end{aligned}
$$

(for the last inequality see [3: Chapter IV/Exercise 2.12]) and

$$
\sum_{i=1}^{N} \lambda_{i} \gamma_{j}^{2}\left|\tilde{\pi}_{j}^{2}\left(\tau_{i}\right)-\beta_{j} \tilde{\pi}_{j-1}^{2}\left(\tau_{i}\right)\right| \leq \gamma_{j}^{2}\left(\left\|\tilde{\pi}_{j}\right\|_{2}^{2}+\beta_{j}\left\|\tilde{\pi}_{j-1}\right\|_{2}^{2}\right)=2 .
$$

The sums containing derivatives can be similarly estimated using $\left\|\tilde{\pi}_{j}^{\prime}\right\|_{2} \leq C j^{\alpha}\left\|\tilde{\pi}_{j}\right\|_{2}=$ $C j^{\alpha} \gamma_{j}^{-1}$, and we arrive at our proposition

Corollary 2. Let $\sigma_{N}=\sum_{i=1}^{N} \lambda_{i} \delta_{\tau_{i}}$ be a discrete measure satisfying

$$
\lambda_{i}^{-1} \leq C_{1} N^{\alpha} \quad \text { and } \quad \frac{1}{\left|\tau_{i}-\tau_{j}\right|} \leq C_{2} N^{\beta} \quad(i \neq j \in\{1, \ldots, N\}) .
$$

Then we have the estimate

$$
\text { cond } H_{n} \leq C_{3}\left(C_{1}, C_{2}, \alpha, \beta\right) N^{\alpha / 2+\beta+1} \quad \text { for } n \leq N
$$

where $C_{3}\left(C_{1}, C_{2}, \alpha, \beta\right)$ denotes a constant depending on $C_{1}, C_{2}$ and $\alpha, \beta$.

Proof. This follows immediately from Theorem 2 and the inequalities in Lemma 13 , since under our assumptions we have $\frac{1+\lambda_{i}^{1 / 2} \lambda_{j}^{-1 / 2}}{\left|r_{i}-\tau_{j}\right|} \leq\left(1+\gamma_{0}^{-1} C_{1}^{1 / 2} N^{\alpha / 2}\right) C_{2} N^{\beta}$ for $i \neq j$ 


\section{Examples}

In this section we compare the performance of the Stieltjes procedure (stielt $j$ ), the stabilized Lanczos process (lancz1 - for technical reasons we used not the original procedure lancz from the ORTHPOL-package, but an own implementation in Pascal based on the Algorithm RKPW in [13: p. 328]) and of the new algorithm (points) based on equations (11) - (14) in a number of examples. In any of these examples we have more or less explicit formulas for the recurrence coefficients and thus we can compute the actual error magnification.

Example 1: Chebyshev measure. We consider the measure

$$
d \sigma(x)=\frac{d x}{\pi \sqrt{1-x^{2}}} \quad \text { on }[-1,1] .
$$

This, of course, is a continuous measure, but the Gauss-Christoffel quadrature formula is well-known in this case. If we set $\tau_{i}=\cos \frac{(2 i-1) \pi}{2 N}$ and $\lambda_{i}=\frac{1}{N}$ for $i=1, \ldots, N$, then the discrete measure $\sigma_{N}=\sum_{i=1}^{N} \lambda_{i} \delta_{\tau_{i}}$ is a discretization of algebraic precision $2 N-1$ and consequently $\mathbf{b}_{n}(\sigma)=\mathbf{b}_{n}\left(\sigma_{N}\right)$ for $n \leq N$. The coefficients $\mathbf{b}_{n}$ are known explicitly in this example. We have $\alpha_{n} \equiv 0$ due to symmetry of the measure, and

$$
\beta_{n}=\left\{\begin{array}{ll}
\frac{1}{2} & \text { for } n=1 \\
\frac{1}{4} & \text { for } n \geq 2
\end{array} \quad \text { and } \quad \gamma_{n}^{2}= \begin{cases}1 & \text { for } n=0 \\
2^{2 n-1} & \text { for } n \geq 1\end{cases}\right.
$$

Table 1: Error magnification in $\mathbf{b}_{n}$ for Example 1, $N=50$

\begin{tabular}{r|c|c|c}
$n$ & points & lancz1 & stielt $j$ \\
\hline 10 & $9.00 \mathrm{e}+00$ & $6.00 \mathrm{e}+00$ & $4.00 \mathrm{e}+00$ \\
20 & $1.40 \mathrm{e}+01$ & $1.00 \mathrm{e}+01$ & $1.00 \mathrm{e}+01$ \\
30 & $3.40 \mathrm{e}+01$ & $3.20 \mathrm{e}+01$ & $1.50 \mathrm{e}+01$ \\
40 & $3.40 \mathrm{e}+01$ & $3.20 \mathrm{e}+01$ & $2.80 \mathrm{e}+01$ \\
50 & $3.40 \mathrm{e}+01$ & $4.00 \mathrm{e}+01$ & $2.80 \mathrm{e}+01$ \\
\hline
\end{tabular}

The orthogonal polynomials are well-known, too. The monic polynomials are

$$
\tilde{\pi}_{n}=2^{-n+1} T_{n} \quad \text { and } \quad \tilde{\rho}_{n}=2^{-n+1} U_{n-1},
$$

where $T_{n}$ and $\dot{U}_{n}$ denote the Chebyshev polynomials of first and second kind, respectively. From this we can calculate easily

$$
\text { cond } H_{n}=O(n)
$$

uniformly in $N$. Thus our problem is well-conditioned, but we have to estimate the quantities characterizing the stability of the algorithms stieltj and points. Since

$$
E_{n}=\varepsilon^{2} \dot{\gamma}_{n}^{2} \int \tilde{\rho}_{n}^{2}(x) d \sigma(x)=2 \varepsilon^{2} n
$$


the Stieltjes procedure is expected to be stable. The same is true for the new algorithm points, if points are added in ascending order: The stability is characterized by the bounds in Lemma 9, which grow only polynomially in $N$.

The results of numerical computations are shown in Table 1. The error magnification tabulated there is defined as the maximum of the absolute errors in the $\alpha$; and the relative errors in the $\beta_{j}$, divided by the machine eps (of order $1 \mathrm{E}-19$ ). The figures confirm our expectations concerning the qualitative behaviour of the algorithms.

Example 2: Bessis' example. Following the article of D. Bessis [2] we consider the equilibrium distribution $\sigma$ of the Julia set of the polynomial $T(x)=x^{2}-\lambda$ with $\lambda$ real and $\lambda>2$. This is a Cantor-like set $K \subset[-\xi, \xi]$, where $\xi=\frac{1+\sqrt{1+4 \lambda}}{2}$. Again, it is not a discrete measure, since the support $S(\sigma)=K$ is not finite. But a reasonable discretization is readily found. The measure is characterized by the invariance property

$$
\int f(x) d \sigma(x)=\int \frac{f(-\sqrt{x+\lambda})+f(\sqrt{x+\lambda})}{2} d \sigma(x) .
$$

This property together with the normalization $\sigma([-\infty, \infty])=1$ uniquely defines $\sigma$. Indeed, for a constant function $f \equiv c$ we have $\int f(x) d \sigma(x)=\int c d \sigma(x)=c=f(0)$ due to the normalization. If $f \in \mathcal{P}_{n}$, then

$$
g(x)=\frac{f(-\sqrt{x+\lambda})+f(\sqrt{x+\lambda})}{2}
$$

defines a polynomial $g \in \mathcal{P}_{[n / 2]}$. Consequently, equation (29) recursively defines integrals with respect to measure $\sigma$ for polynomials $f$ of any degree and thus for all continuous functions by continuity. Moreover, we see by induction that for $N=2^{k}$ the set $S_{N}=$ $\left\{\tau_{1}, \ldots, \tau_{N}\right\}=T^{-k}(\{0\})$ of nodes together with the weights $\lambda_{i}=1 / N$ for $i=1, \ldots, N$ give a discretization of algebraic precision $2 N-1$ : If we set $\sigma_{N}=\frac{1}{N} \sum_{\tau \in S_{N}} \delta_{\tau}$ and this is a discretization of algebraic precision $M$, then $\sigma_{2 N}$ has algebraic precision $\geq 2 M+1$ by equation (29). But $\sigma_{1}=\delta_{0}$ has algebraic precision 1 , since $\int x d \sigma(x)=0$ due to symmetry of the measure.

The recurrence coefficients $b_{n}$ are not known explicitly, but can be calculated recursively from the equations

$$
\beta_{1}=\lambda, \quad \beta_{2 k} \beta_{2 k-1}=\beta_{k} \quad \text { and } \quad \beta_{2 k}+\beta_{2 k+1}=\lambda \text { for } k \geq 1
$$

(these are Bessis' Equations [2: Equations IV.105] in our notations). Of course, we have $\alpha_{n} \equiv 0$ due to symmetry. Thus we can compute the actual error magnification in our algorithms. To see what we have to expect we need some estimates of orthogonal polynomials, of their derivatives and of numerator polynomials on $S_{N}$.

On the support of the measure we have explicit bounds for the orthonormal polynomials $\pi_{n}$. Bessis' estimate [2: Inequality IV.141] is

$$
\left|\pi_{n}(x)\right|<\frac{1}{2}\left(\frac{4 \sqrt{\lambda}}{\sqrt{1+4 \lambda}-1}\right)^{k} \quad \text { for } 2^{k-1} \leq n \leq 2^{k}
$$


where $x \in K$. This seems to be of little use, since obviously $S_{N} \cap K=\emptyset$. Fortunately, a careful analysis of the proof shows that estimates of the form

$$
\left|\pi_{n}(x)\right| \leq C(\lambda)^{k} \quad \text { for } 2^{k-1} \leq n \leq 2^{k}
$$

are valid not only for $x \in K$, but also for $x \in T^{-k}([-\xi, \xi])$, and this set contains $T^{-k}(\{0\})=S_{N}$ for $N=2^{k}$. The same is true for the polynomials $\pi_{n}^{\prime}$ and $\rho_{n}$ (we omit technical details here, see the forthcoming paper [6]). Consequently, the condition of our problem can be bounded by a polynomial in $n$ and $N$. This fact we could derive from Corollary 2, too: For $N=2^{k}$ and $S_{N}=\left\{\tau_{1}, \ldots, \tau_{N}\right\}$ we have $\lambda_{i}^{-1}=N$ and

$$
\left|\tau_{i}-\tau_{j}\right| \geq C \cdot(2 \xi)^{-k} \quad \text { for } i \neq j \text {. }
$$

This follows from the definition of $S_{N}$ and the elementary inequality

$$
|\sqrt{\lambda+x}-\sqrt{\lambda+y}| \geq \frac{|x-y|}{2 \sqrt{\lambda+\xi}}=\frac{|x-y|}{2 \xi} \quad \text { for } \quad x, y \in[-\xi, \xi] \text {. }
$$

The estimate (30) together with Lemma 9 shows that the procedure points should be stable. The same is true for the Stieltjes procedure stieltj. The numerical results confirm the expected behaviour of the algorithms.

Table 2: Error magnification in $\mathbf{b}_{n}$ for Example 2, $N=128$

\begin{tabular}{r|c|c|c}
$n$ & points & lancz1 & stieltj \\
\hline 10 & $1.00 \mathrm{e}+01$ & $1.70 \mathrm{e}+01$ & $6.00 \mathrm{e}+00$ \\
20 & $1.40 \mathrm{e}+01$ & $1.70 \mathrm{e}+01$ & $8.00 \mathrm{e}+00$ \\
30 & $2.40 \mathrm{e}+01$ & $1.70 \mathrm{e}+01$ & $1.60 \mathrm{e}+01$ \\
40 & $2.60 \mathrm{e}+01$ & $1.80 \mathrm{e}+01$ & $3.30 \mathrm{e}+01$ \\
50 & $3.80 \mathrm{e}+01$ & $4.70 \mathrm{e}+01$ & $3.40 \mathrm{e}+01$ \\
128 & $9.70 \mathrm{e}+01$ & $1.09 \mathrm{e}+02$ & $8.20 \mathrm{e}+01$ \\
\hline
\end{tabular}

Example 3: An atomic measure with a dense set of mass points. In the preceding example we assumed $\lambda>2$ and obtained a singular measure concentrated on a Cantor-type set $K$ of Lebesgue measure 0 . For the "degenerate" case $\lambda=2$ we would obtain an absolutely continuous measure on $[-2,2]-$ in fact this is simply a Chebyshev measure scaled to this interval. If we modify the defining invariance property (29), then we have a more interesting example: Let the measure $\sigma$ with support $S(\sigma)=[-2,2]$ be defined by

$$
\int f(x) d \sigma(x)=\int \frac{f(-\sqrt{x+2})+f(0)+f(\sqrt{x+2})}{3} d \sigma(x)
$$

and the normalization $\int 1 d \sigma(x)=\sigma([-2,2])=\gamma_{0}^{-2}=1$. Clearly, this measure puts mass $3^{-k-1}$ on the points $2 \cos (2 m-1) 2^{-k-1} \pi$ for $k \geq 0$ and $m=1,2, \ldots, 2^{k}$. This is an infinite set of points dense in $[-2,2]$. Again, it is easy to find a suitable discretization 
of the measure. The measure $\sigma_{1}=\delta_{0}$ is a discretization of algebraic precision 1. If we are given a discretization $\sigma_{N}$ of algebraic precision $M$, then we can construct a new discretization

$$
\sigma_{2 N+1}=\frac{1}{3} \delta_{0}+\sum_{\tau^{2}-2 \in S_{N}} \frac{1}{3} \sigma_{N}\left(\left\{\tau^{2}-2\right\}\right) \delta_{\tau}
$$

with algebraic precision $2 M+1$ using the invariance (31). Thus by induction we obtain discretizations $\sigma_{N}$ of algebraic precision $N$ with $N=2^{k}-1$.

The recurrence coefficients $b_{n}$ can be calculated from a system of equations similar to (though more complicated than) the equations in the preceding example. The following proposition will be given without proof (for details see [6]).

Proposition 2. Let $\nu$ be measure $\sigma$ without the point mass at 0 , i.e. $\nu(B)=$ $\sigma(B \backslash\{0\})$ for any measurable set $B$ on the real line. We have $\alpha_{n}(\sigma) \equiv 0$ due to symmetry of the measure. The coefficients $\beta_{n}(\sigma)$ can be calculated from

$$
\beta_{1}(\nu)=2, \quad \beta_{2 n}(\nu)+\beta_{2 n+1}(\nu)=2, \quad \beta_{2 n}(\nu) \beta_{2 n-1}(\nu)=q_{n} \beta_{n}(\nu)=\beta_{n}(\sigma)
$$

for $n \geq 1$, where

$$
q_{n}=\left\{\begin{array}{ll}
\frac{2}{3} & \text { for } n=1 \\
\frac{1+\frac{1}{2}\left(1+s_{k}\right)}{1+\frac{1}{2}\left(1+s_{k-1}\right)} & \text { for } n=2 k \\
\frac{1}{q_{n-1}} & \text { for } n=2 k+1
\end{array}, \quad s_{k}=\sum_{l=1}^{k} \frac{\beta_{1}(\nu) \cdots \beta_{2 l-1}(\nu)}{\beta_{2}(\nu) \cdots \beta_{2 l}(\nu)}\right.
$$

for $k \geq 1$

Table 3: Error magnification in $\mathbf{b}_{n}$ for Example $3, N=127$

\begin{tabular}{c|c|c|c}
$n$ & points & lancz1 & stieltj \\
\hline 10 & $2.80 \mathrm{e}+01$ & $2.20 \mathrm{e}+01$ & $3.00 \mathrm{e}+01$ \\
20 & $4.50 \mathrm{e}+01$ & $4.60 \mathrm{e}+01$ & $3.00 \mathrm{e}+01$ \\
30 & $4.50 \mathrm{e}+01$ & $4.60 \mathrm{e}+01$ & $3.00 \mathrm{e}+01$ \\
40 & $5.50 \mathrm{e}+01$ & $4.60 \mathrm{e}+01$ & $3.00 \mathrm{e}+01$ \\
50 & $5.50 \mathrm{e}+01$ & $4.60 \mathrm{e}+01$ & $3.00 \mathrm{e}+01$ \\
\hline
\end{tabular}

Estimates for derivatives of orthogonal polynomials are readily available in this example, since $S=[-2,2]$ preserves Markov's inequality: In the notations of the Appendix we have $S \in \mathcal{M}^{\infty}\left(\frac{1}{2}, 2\right)$, and obviously our measure $\sigma$ satisfies condition (34) with $s=$ $\ln 3 / \ln 2$. Consequently, $S$ preserves Markov's inequality in $L^{2}(\sigma)$, i.e. $S \in \mathcal{M}^{2}(M, 2+$ $s)$, due to [14: Proposition 1]. Thus, our problem is well-conditioned due to Corollary 1 , and the procedure stieltj is stable in virtue of Lemma 8 . The assumptions of Corollary 2 are satisfied as well (with $\alpha=\ln 3 / \ln 2$ and $\beta=2$ ), and the procedure points is stable due to Lemma 9 . The numerical results in Table 3 agree with these expectations (observe that the algebraic precision of our discretization $\sigma_{N}$ is only $N$ for $N=2^{k}-1$ and thus we can calculate only the recursion coefficients $\mathbf{b}_{n}$ with $\left.2 n-1 \leq N\right)$. 
Example 4: Chebyshev measure plus point mass. In this example we use the measure $\sigma=\sigma_{c}+\delta_{2}$, where the measure $\sigma_{c}$ is defined by

$$
d \sigma_{c}(x)=\frac{d x}{\pi \sqrt{1-x^{2}}} \text { on }[-1,1]
$$

i.e. we add a point mass of weight 1 at $t=2$ to the measure from Example 1. The recurrence coefficients can be calculated easily from the formulas of Section 3 . We obtain $\gamma_{0}^{2}=1 / 2$ and

$$
\gamma_{j}^{2}=2^{2 j-1} \frac{U_{2 j-2}(2)+2 j+1}{U_{2 j}(2)+2 j+3} \quad \text { for } j \geq 1 .
$$

The coefficients $\beta_{j}$ can be computed from equation (7), and the coefficients $\alpha_{j}$ from the equation

$$
\sum_{i=0}^{j} \alpha_{i}=\frac{2 T_{j}(2) T_{j+1}(2)}{U_{2 j}(2)+2 j+3}
$$

A discretization of the measure can be obtained trivially from the discretization $\sigma_{N-1}$ in Example 1 by adding $\lambda_{N}=1$ and $\tau_{N}=2$ (and consequently the algebraic precision of $\sigma_{N}$ is only $2 N-3$, i.e. we can calculate $b_{n}$ for $n \leq N-1$ ). The assumptions of Corollary 2 are satisfied, and thus our problem is well-conditioned. The procedure points should be stable by Lemma 9 . In the Stieltjes procedure stieltj, however, we can expect instability: The inequalities in Lemmas 11 and 12 show that the ratio $\tilde{\rho}_{j}(2) / \tilde{\pi}_{j}(2)$ grows exponentially (in fact like $\left.(2+\sqrt{3})^{2 j}=(7+4 \sqrt{3})^{j}\right)$. The data in Table 4 indeed show this behaviour. We mention here that instability of the Stieltjes procedure in this situation was reported by Gautschi (compare [8: Example 4.8]).

Table 4: Error magnification in $\mathbf{b}_{n}$ for Example $4, N=80$

\begin{tabular}{c|c|c|c}
$n$ & points & lancz1 & stieltj \\
\hline 10 & $5.20 e+01$ & $4.80 e+01$ & $5.53 e+01$ \\
20 & $1.02 e+02$ & $1.04 e+02$ & $1.08 e+02$ \\
30 & $1.56 e+02$ & $1.58 e+02$ & $1.05 e+13$ \\
40 & $2.32 e+02$ & $2.28 e+02$ & $4.45 e+19$ \\
50 & $2.32 e+02$ & $2.28 e+02$ & $4.45 e+19$ \\
79 & $4.66 e+02$ & $4.76 e+02$ & $5.13 e+19$ \\
\hline
\end{tabular}

Example 5: Discrete Chebyshev polynomials. These polynomials are orthogonal with respect to the discrete measure

$$
\sigma_{N}=\frac{1}{N} \sum_{i=1}^{N} \delta_{i-1}
$$

The recurrence coefficients are known explicitly: We have

$$
\alpha_{k}=\frac{N-1}{2}(k=0,1, \ldots, N-1) \text { and } \beta_{k}=\frac{1-(k / N)^{2}}{4\left(1-1 / k^{2}\right)}(k=1, \ldots, N-1) .
$$


The values of the orthonormal polynomials $\pi_{n}$ at 0 can be evaluated easily using the explicit formulas in [1: Section 22.19]. After some calculation we obtain

$$
\pi_{n}(0)=(-1)^{n}(N-1) ! \sqrt{\frac{(2 n+1) N}{(N+n) !(N-n-1) !}} \quad \text { for } n=0, \ldots, N-1 \text {. }
$$

Now from equation (33) there follows

$$
\frac{\tilde{\rho}_{n+1}(0)}{\tilde{\pi}_{n+1}(0)}-\frac{\tilde{\rho}_{n}(0)}{\tilde{\pi}_{n}(0)}=\frac{1}{\gamma_{n}^{2} \tilde{\pi}_{n}(0) \tilde{\pi}_{n+1}(0)}=\frac{1}{\beta_{n+1}^{1 / 2} \pi_{n}(0) \pi_{n+1}(0)},
$$

since we have $\gamma_{0}^{2}=1$. This gives

$$
\frac{\tilde{\rho}_{n}(0)}{\tilde{\pi}_{n}(0)}=\sum_{l=1}^{n} \frac{1}{\beta_{l}^{l / 2} \pi_{l-1}(0) \pi_{l}(0)}=-2 \sum_{l=1}^{n} \frac{(N+l-1) !(N-l-1) !}{(N-1) !^{2}}
$$

and this quantity grows very rapidly as $n$ approaches $N$ (for $n=N-1$ its absolute value is of order $2^{2 N}$ ). Consequently, we can expect instable behaviour of the Stieltjes procedure. The problem is well-conditioned, however, and the new algorithm points should be stable, as the estimates in Lemma 9 and Corollary 2 show.

The numerical results reported in Table 5 confirm our expectations (the instability of the Stieltjes procedure in this case was observed already by Gautschi, see [8: Example 4.1]).

Table 5: Error magnification in $b_{n}$ for Example 5, $N=80$

\begin{tabular}{c|c|c|c}
$n$ & points & lancz1 & stieltj \\
\hline 60 & $2.80 e+01$ & $4.70 e+01$ & $4.00 e+01$ \\
65 & $2.90 e+01$ & $6.00 e+01$ & $9.44 e+05$ \\
70 & $4.70 e+01$ & $6.00 e+01$ & $1.49 e+11$ \\
75 & $8.00 e+01$ & $6.00 e+01$ & $3.36 e+17$ \\
80 & $1.24 e+02$ & $6.00 e+01$ & $5.05 e+20$ \\
\hline
\end{tabular}

\section{Appendix}

In this section we summarize some estimates used for the proofs of our results.

Definition 5. Let $\tilde{\pi}_{j}$ be the monic orthogonal polynomials with respect to some measure $\sigma$, satisfying the recurrence relation (2). The (monic)numeratorpolynomials $\tilde{\rho}_{j}$ are defined by

$$
\tilde{\rho}_{j}(x)=\gamma_{0}^{2} \int \frac{\tilde{\pi}_{j}(x)-\tilde{\pi}_{j}(t)}{x-t} d \sigma(t) .
$$

These polynomials (which are called sometimes associated polynomials or polynomials of second kind) satisfy the same recurrence relation (2), except for $j=0$ (if we do not make special assumptions about $\beta_{0}$ ). Note, however, that $\tilde{\rho}_{j}$ is a monic polynomial of degree $j-1$ (compare the different notation in [3]). We will use the following simple property of numerator polynomials (this is just [3: Chapter III/Identity (4.4)] in our notations). 
Lemma 6. The identity

$$
\tilde{\pi}_{n}(x) \tilde{\rho}_{n+1}(x)-\tilde{\pi}_{n+1}(x) \tilde{\rho}_{n}(x)=\frac{\gamma_{0}^{2}}{\gamma_{n}^{2}}
$$

holds for $n \geq 0$.

In sufficiently regular cases the numerator polynomials grow not essentially faster than the orthogonal polynomials. This depends on the measure and its support. The following definition is taken from [14].

Definition 6. Let $1 \leq p \leq \infty$, let $S \subset \mathbb{R}$ be a compact set and let $\sigma$ be a measure with support $S$ satisfying, for some constants $c_{0}>0$ and $s>0$,

$$
\sigma(B(x, r)) \geq c_{0} r^{s} \quad(x \in S, 0<r \leq 1)
$$

where $B(x, r)$ denotes the closed ball with center $x$ and radius $r$. The set $S$ preserves Markov's incquality in $L^{p}(\sigma)$ if there exist constants $M>0$ and $\alpha>0$ such that

$$
\left\|q^{\prime}\right\|_{p} \leq M n^{\alpha}\|q\|_{p} \quad \text { for } \quad q \in \mathcal{P}_{n}
$$

(and for this we write $S \in \mathcal{M}^{p}(M, \alpha)$ ). The norm $\|\cdot\|_{p}$ is defined as usually: $\|q\|_{p}=$ $\left(\int|q(x)|^{p} d \sigma(x)\right)^{1 / p}$ for $p<\infty$ and $\|q\|_{\infty}=\sup _{x \in S}|q(x)|$.

For our purposes, of course, only the cases $p=2$ and $p=\infty$ are of interest. In order to estimate numerator polynomials, we need the division inequality [14: Proposition 2].

Lemma 7. Let $1 \leq p \leq \infty$, let $S \in \mathcal{M}^{p}(M, \alpha)$ and let $q$ be a polynomial in $\mathcal{P}_{n}$ of the form $q(x)=\left(x-x_{0}\right) q_{1}(x)$, where $x_{0} \in \mathbb{R}$. Then $\left\|q_{1}\right\|_{p} \leq 6 M n^{\alpha}\|q\|_{p}$.

Lemma 8. Let $\sigma$ be a measure with $S(\sigma) \in \mathcal{M}^{2}(M, \alpha)$, and let $\tilde{\pi}_{n}$ and $\tilde{\rho}_{n}$ be the monic orthogonal and numerator polynomials, respectively. Then $\frac{\left\|\tilde{\rho}_{n}\right\|_{2}^{2}}{\left\|\tilde{\pi}_{n}\right\|_{2}^{2}} \leq 72 M^{2} n^{2 \alpha}$.

Proof. For fixed $x$, we set $q(t)=\tilde{\pi}_{n}(x)-\tilde{\pi}_{n}(t)=(x-t) q_{1}(t)$. Then we have from

$$
\left|\tilde{\rho}_{n}(x)\right| \leq \gamma_{0}^{2}\left\|q_{1}\right\|_{1} \leq \gamma_{0}\left\|q_{1}\right\|_{2} \leq 6 M n^{\alpha} \gamma_{0}\|q\|_{2}
$$

and (if $n>0$ ) $\|q\|_{2}^{2}=\gamma_{0}^{-2} \tilde{\pi}_{n}^{2}(x)+\gamma_{n}^{-2}$. Thus, we obtain $\tilde{\rho}_{n}^{2}(x) \leq 36 M^{2} n^{2 \alpha}\left[\tilde{\pi}_{n}^{2}(x)+\right.$ $\left.\gamma_{0}^{2} \gamma_{n}^{-2}\right]$ and consequently $\left\|\tilde{\rho}_{n}^{2}\right\|_{2}^{2} \leq 72 M^{2} n^{2 \alpha} \gamma_{n}^{-2}=72 M^{2} n^{2 \alpha}\left\|\tilde{\pi}_{n}^{2}\right\|_{2}^{2}$

The result, of course, is applicable only to discretizations of continuous measures (since finite sets trivially do not preserve Markov's property).

Outside the interval of orthogonality the numerator polynomials grow not faster than the orthogonal polynomials.

Lemma 9. Let $\sigma=\sum_{i=1}^{N} \lambda_{i} \delta_{r_{i}}$ be a discrete measure with support $S(\sigma)=\left\{\tau_{1}, \ldots\right.$, $\left.\tau_{N}\right\} \subset[a, b]$ and let $r$ be a point with $\tau>b$. Then we have the estimate

$$
\frac{\tilde{\rho}_{n}(\tau)}{\tilde{\pi}_{n}(\tau)} \leq \gamma_{0}^{2} \sum_{i=1}^{N} \frac{\lambda_{i}}{\tau-\tau_{i}} \quad \text { for } n \leq N
$$


Proof. Since all zeros of $\tilde{\pi}_{n}$ and $\tilde{\rho}_{n}$ are in the interval $[a, b]$ and the polynomials are monic, they are positive for $\tau>b$. Now from (33) the inequality

$$
\frac{\tilde{\rho}_{n}(\tau)}{\tilde{\pi}_{n}(\tau)}<\frac{\tilde{\rho}_{n+1}(\tau)}{\tilde{\pi}_{n+1}(\tau)} \quad \text { for } n+1 \leq N
$$

follows immediately. Thus we have

$$
\frac{\tilde{\rho}_{n}(\tau)}{\tilde{\pi}_{n}(\tau)} \leq \frac{\tilde{\rho}_{N}(\tau)}{\tilde{\pi}_{N}(\tau)}=\gamma_{0}^{2} \sum_{i=1}^{N} \frac{\lambda_{i}}{\tau-\tau_{i}}
$$

by the definition of numerator polynomials, since $\tilde{\pi}_{N}\left(\tau_{i}\right)=0$

However, in isolated points of the support the behavior of orthogonal polynomials and numerator polynomials changes - the first show exponential decay, the latter exponential growth.

Definition 7. The distance of a point $x \in \mathbb{R}$ from a set $S \subset \mathbb{R}$ will be denoted by $d(x, B)$, i.e. $d(x, B)=\inf _{y \in B}|x-y|$.

Lemma 10. Let $\sigma$ be a measure with bounded support $S(\sigma) \subset[a, b]$ and let $\tau$ be a point with $d(\tau, S(\sigma)) \geq \delta$. Then there exists a number $c(\tau)>1$ with

$$
\sum_{i=0}^{n} \gamma_{i}^{2} \tilde{\pi}_{i}^{2}(\tau) \geq \frac{\gamma_{0}^{2}}{4} c(\tau)^{n-1}
$$

Proof. In terms of the orthonormal polynomials $\pi_{i}$ the left-hand side of our inequality is $\sum_{i=0}^{n} \pi_{i}^{2}(\tau)$. But its reciprocal value is the Christoffel function

$$
\cdot\left(\sum_{i=0}^{n} \pi_{i}^{2}(\tau)\right)^{-1}=\min _{q \in \mathcal{P}_{n}} \frac{\int q^{2}(x) d \sigma(x)}{q^{2}(\tau)}
$$

(sce [3: Chapter I/Theorem 7.3] or [16: p. 4]). Now we construct a polynomial $q \in \mathcal{P}_{n}$ with $|q| \leq 1$ on $S(\sigma)$. If we set

$$
M(\tau)=\max \left\{(a-\tau)^{2},(b-\tau)^{2}\right\}
$$

then we have $\delta^{2} \leq(x-\tau)^{2} \leq M(\tau)$ for any $x \in S(\sigma)$, and from this there follows

$$
-1 \leq 2 \frac{(x-\tau)^{2}-\delta^{2}}{M(\tau)-\delta^{2}}-1 \leq 1
$$

on $S(\sigma)$. Consequently, the polynomial

$$
q(x)=T_{\left[\frac{n}{2}\right]}\left(2 \frac{(x-\tau)^{2}-\delta^{2}}{M(\tau)-\delta^{2}}-1\right)
$$


has the desired properties. We see easily

$$
\int q^{2}(x) d \sigma(x) \leq \int 1 d \sigma(x)=\gamma_{0}^{-2} \quad \text { and } \quad q^{2}(\tau)=T_{\left[\frac{n}{2}\right]}^{2}\left(\frac{2 \delta^{2}}{M(\tau)-\delta^{2}}+1\right)
$$

The explicit expression

$$
T_{m}(x)=\frac{1}{2}\left[\left(x+\sqrt{x^{2}-1}\right)^{m}+\left(x-\sqrt{x^{2}-1}\right)^{m}\right]
$$

(this is [15: Equation 4.13]) shows that

$$
q^{2}(\tau) \geq \frac{1}{4}\left(x(\tau)+\sqrt{x(\tau)^{2}-1}\right)^{2\left[\frac{n}{2}\right]} \geq \frac{1}{4}\left(x(\tau)+\sqrt{x(\tau)^{2}-1}\right)^{n-1}
$$

with $x(\tau)=\frac{2 \delta^{2}}{M(\tau)-\delta^{2}}+1>1$. From (35) - (37) our proposition with $c(\tau)=x(\tau)+$ $\sqrt{x(\tau)^{2}-1}$ follows immediately

Lemma 11. Let $\nu$ be a measure with bounded support. $S(\nu) \subset[a, b]$ and let $\tau$ be $a$ point with $\nu(\{\tau\})=\lambda>0$ and $d(\tau, S(\nu) \backslash\{\tau\}) \geq \delta$. Let $p_{j}$ be the monic orthogonal polynomials with respect to $\nu$. Then there exists a number $c(\tau)>1$ with

$$
\gamma_{n}(\nu)^{2} p_{n}^{2}(\tau) \leq \frac{1}{\lambda\left(1+\frac{1}{4} \lambda \gamma_{0}(\nu)^{2} c(\tau)^{n-2}\right)} .
$$

Proof. Under our assumptions $\sigma=\nu-\lambda \delta_{r}$ is a measure with support $S(\sigma)=$ $S(\nu) \backslash\{\tau\}$ and we have $d(\tau, S(\sigma)) \geq \delta$. Thus, the assumptions of Lemma 10 are fulfilled, and the monic orthogonal polynomials $\tilde{\pi}_{j}$ with respect to $\sigma$ satisfy the inequality there. The measure $\nu$ is obtained from the measure $\sigma$ by adding a point mass at $\tau$ of weight $\lambda$. Now from equations (16) and (19) we obtain easily

$$
\begin{aligned}
\gamma_{n}(\nu)^{2} p_{n}^{2}(\tau) & =\gamma_{n}^{2} \frac{1+\lambda \sum_{i=0}^{n-1} \gamma_{i}^{2} \tilde{\pi}_{i}^{2}(\tau)}{1+\lambda \sum_{i=0}^{n} \gamma_{i}^{2} \tilde{\pi}_{i}^{2}(\tau)}\left[\frac{\tilde{\pi}_{n}(\tau)}{1+\lambda \sum_{i=0}^{n-1} \gamma_{i}^{2} \tilde{\pi}_{i}^{2}(\tau)}\right]^{2} \\
& =\frac{1}{\lambda}\left(\frac{1}{1+\lambda \sum_{i=0}^{n-1} \gamma_{i}^{2} \tilde{\pi}_{i}^{2}(\tau)}-\frac{1}{1+\lambda \sum_{i=0}^{n} \gamma_{i}^{2} \tilde{\pi}_{i}^{2}(\tau)}\right) \\
& \leq \frac{1}{\lambda\left(1+\lambda \sum_{i=0}^{n-1} \gamma_{i}^{2} \tilde{\pi}_{i}^{2}(\tau)\right)}
\end{aligned}
$$

Our proposition follows from Lemma 10, since $\gamma_{0}(\nu)^{2}=\frac{\gamma_{0}^{2}}{1+\lambda \gamma_{0}^{2}} \leq \gamma_{0}^{2}$ 
Lemma 12. Let $\sigma$ be a measure with bounded support $S(\sigma) \subset[a, b]$ and let $\tau$ be $a$ point with $\sigma(\{\tau\})=\lambda>0$ and $d(\tau, S(\sigma) \backslash\{\tau\}) \geq \delta$. Let $\tilde{\rho}_{j}$ be the monic numerator polynomials with respect to $\sigma$. Then there exists a number $c(\tau)>1$ with

$$
\max \left\{\gamma_{n}^{2} \tilde{\rho}_{n}^{2}(\tau), \gamma_{n+1}^{2} \tilde{\rho}_{n+1}^{2}(\tau)\right\} \geq \frac{\lambda \gamma_{0}^{4}}{(b-a)^{2}}\left(1+\frac{1}{4} \lambda \gamma_{0}^{2} c(\tau)^{n-2}\right) .
$$

Proof. From equation (33) we have

$$
\gamma_{n} \gamma_{n+1}\left[\tilde{\pi}_{n}(x) \tilde{\rho}_{n+1}(x)-\tilde{\pi}_{n+1}(x) \tilde{\rho}_{n}(x)\right]=\gamma_{0}^{2} \frac{\gamma_{n+1}}{\gamma_{n}}=\gamma_{0}^{2} \beta_{n+1}^{-1 / 2}>\frac{2 \gamma_{0}^{2}}{b-a}
$$

where we used the well-known fact that

$$
\beta_{n}<\frac{(b-a)^{2}}{4}
$$

(see [3: Chapter IV/Exercise 2.12]). Consequently, the inequality

$$
\max \left\{\left|\gamma_{n} \gamma_{n+1} \tilde{\pi}_{n}(x) \tilde{\rho}_{n+1}(x)\right|,\left|\gamma_{n} \gamma_{n+1} \tilde{\pi}_{n+1}(x) \tilde{\rho}_{n}(x)\right|\right\}>\frac{\gamma_{0}^{2}}{b-a}
$$

holds. Squaring both sides and using inequality (11) for the measure $\sigma$ we obtain our proposition

The estimate of the condition of our problem given in Theorem 2 requires information about the size of orthogonal polynomials and their derivative in the nodes $\tau_{i}$. But even if our discrete measure is the discretization of some continuous measure with support $S$ preserving Markov's inequality, this may be not very helpful, since the nodes are not necessarily contained in $S$. For this reason we give some rough estimates based on the discretization itself.

Lemma 13. Let $\sigma=\sum_{i=1}^{N} \lambda_{i} \delta_{\tau_{i}}$ be a discrete measure with $S(\sigma) \subset\{a, b]$ and $\tilde{\pi}_{n}$ the monic orthogonal polynomials with respect to $\sigma$. Then the estimates

$$
\begin{aligned}
&\left|\lambda_{j} \gamma_{n}^{2} \tilde{\pi}_{n}^{2}\left(\tau_{j}\right)\right| \leq 1 \\
&\left|\lambda_{j} \gamma_{n}^{2} \tilde{\pi}_{n}\left(\tau_{j}\right) \tilde{\pi}_{n+1}^{\prime}\left(\tau_{j}\right)\right| \leq 1+\frac{b-a}{2} \sum_{i \neq j} \frac{1+\lambda_{i}^{1 / 2} \lambda_{j}^{-1 / 2}}{\left|\tau_{i}-\tau_{j}\right|} \\
&\left|\lambda_{j} \gamma_{n}^{2} \tilde{\pi}_{n+1}\left(\tau_{j}\right) \tilde{\pi}_{n}^{\prime}\left(\tau_{j}\right)\right| \leq \frac{b-a}{2} \sum_{i \neq j} \frac{1+\lambda_{i}^{1 / 2} \lambda_{j}^{-1 / 2}}{\left|\tau_{i}-\tau_{j}\right|} \\
&\left|\lambda_{j} \gamma_{n}^{2} \tilde{\pi}_{n}\left(\tau_{j}\right) \tilde{\pi}_{n}^{\prime}\left(\tau_{j}\right)\right| \leq \sum_{i \neq j} \frac{1+\lambda_{i}^{1 / 2} \lambda_{j}^{-1 / 2}}{\left|\tau_{i}-\tau_{j}\right|}
\end{aligned}
$$

hold.

Proof. The first estimate follows easily from the obvious equation

$$
\gamma_{n}^{-2}=\int \tilde{\pi}_{n}^{2}(x) d \sigma(x)=\sum_{i=1}^{N} \lambda_{i} \tilde{\pi}_{n}^{2}\left(\tau_{i}\right) \geq \lambda_{j} \tilde{\pi}_{n}^{2}\left(\tau_{j}\right)
$$


Now we prove the second estimate: The polynomial $\frac{\bar{\pi}_{n+1}(x)-\bar{\pi}_{n+1}\left(r_{j}\right)}{x-\tau_{j}} \in \mathcal{P}_{n}$ is monic and thus we have

$$
\int \frac{\tilde{\pi}_{n+1}(x)-\tilde{\pi}_{n+1}\left(\tau_{j}\right)}{x-\tau_{j}} \tilde{\pi}_{n}(x) d \sigma(x)=\gamma_{n}^{-2}
$$

This equation can be written as

$$
\lambda_{j} \tilde{\pi}_{n+1}^{\prime}\left(\tau_{j}\right) \tilde{\pi}_{n}\left(\tau_{j}\right)+\sum_{i \neq j} \lambda_{i} \frac{\tilde{\pi}_{n+1}\left(\tau_{i}\right)-\tilde{\pi}_{n+1}\left(\tau_{j}\right)}{\tau_{i}-\tau_{j}} \tilde{\pi}_{n}\left(\tau_{i}\right)=\gamma_{n}^{-2}
$$

From our first estimate we obtain $\left|\gamma_{n} \tilde{\pi}_{n}\left(\tau_{j}\right)\right| \leq \lambda_{j}^{-1 / 2}$ and

$$
\left|\gamma_{n} \tilde{\pi}_{n+1}\left(\tau_{j}\right)\right| \leq \frac{\gamma_{n}}{\gamma_{n+1}} \lambda_{j}^{-1 / 2}=\beta_{n}^{1 / 2} \lambda_{j}^{-1 / 2} \leq \frac{b-a}{2} \lambda_{j}^{-1 / 2}
$$

using (38). Substituting these inequalities into (39) we arrive at our proposition. Starting from

$$
\int \frac{\tilde{\pi}_{n}(x)-\tilde{\pi}_{n}\left(\tau_{j}\right)}{x-\tau_{j}} \tilde{\pi}_{n}(x) d \sigma(x)=0 \quad \text { and } \quad \int \frac{\tilde{\pi}_{n}(x)-\tilde{\pi}_{n}\left(\tau_{j}\right)}{x-\tau_{j}} \tilde{\pi}_{n+1}(x) d \sigma(x)=0
$$

the last two inequalities can be shown analogously

\section{References}

[1] Abramowitz, M. and I. Stegun (eds.): Pocketbook of Mathematical Functions (Abridget edition of: Handbook of Mathematical Functions). Thun/Frankfurt am Main: Verlag Harri Deutsch 1984.

[2] Bessis, D.: Orthogonal polynomials, Padé approximations and Julia sets. In: Orthogonal Polynomials, Theory and Practice (NATO ASI Series C: Vol. 294; ed.: P. Nevai). Dordrecht - Boston - London: Kluwer Acad. Publ. 1990, pp. 55 - 97.

[3] Chihara, 'T. S.: An Introduction to Orthogonal Polynomials. New York: Gordon \& Breach 1978.

[4] Fischer, H.-J.: Recurrence coefficients of orthogonal polynomials with respect to some selfsimilar singular distributions. Z. Anal. Anw. 14 (1995), $141-155$.

[5] Fischer, H.-.].: On the condition of orthogonal polynomials via modified moments. Z. Anal. Anw. 15 (1996), 223 - 244.

[6] Fischer, H.-J.: On generating orthogonal polynomials for self-similar measures. In preparation.

[7] Gautschi, W.: Questions of numerical condition related to polynomials. In: Recent Advances in Numerical Analysis (eds.: C. de Boor and G. H. Golub). New York: Acad. Press 1978.

[8] Gautschi, W.: On generating orthogonal polynomials. SIAM J. SCI. STAT. COMP. 3 (1982), $289-317$.

[9] Gautschi, W.: On the sensitivity of orthogonal polynomials to perturbations in the mo. ments. Numer. Math. 48 (1986), $369-382$. 
[10] Gautschi, W.: Computational aspects of orthogonal polynomials. In: Orthogonal Polynomials, Theory and Practice (NATO ASI Series C: Vol. 294; ed.: P. Nevai). Dordrecht Boston - London: Kluwer Acad. Publ. 1990, pp. $181-216$.

[11] Gautschi, W.: Is the recurrence relation for orthogonal polynomials always stable? BIT 33 (1993), 277 - 284.

[12] Gautschi, W.: Algorithm 726: ORTHPOL - A package of routines for generating orthogonal polynomials and Gauss-type quadrature rules. ACM Trans. Math. Software 20 (1994), 21 - 62 .

[13] Gragg, W. B. and W. J. Harrod: The numerically stable reconstruction of Jacobi matrices from spectral data. Numer. Math. 44 (1984), 317 - 335.

[14] Jonsson, A.: Markov's inequality and zeros of orthogonal polynomials on fractal sets. J. Approx. Theory 78 (1994), 87 - 97.

[15] Meinardus, G.: Approximation von Funktionen und ihre numerische Behandlung. Berlin et al.: Springer-Verlag 1964.

[16] Nevai, P.: Orthogonal Polynomials Mem. Amer. Math Soc. 18 (1979), No. 213.

[17] Reichel, L.: Construction of polynomials that are orthogonal with respect to a discrete bilinear form. Adv. Comput. Math. 1 (1993), $241-258$.

Received 10.02.1997 